\title{
La « démarche-action Montréal-nord ", une approche territoriale visant à développer des interventions intégrées
}

\author{
Danièle Aveline, Ministère des Affaires municipales et des Régions ${ }^{1}$ \\ André Bergeron, Direction de Santé publique de Montréal
}

\section{Introduction}

$\sum$ a situation des personnes défavorisées à Montréal est connue. Plusieurs secteurs de la ville comptent des proportions importantes de personnes vivant sous le seuil de faible revenu. Face à cet état de choses, la Conférence administrative régionale - Développement social (CAR-DS), qui regroupe les directions régionales des ministères à vocation sociale et économique, souhaitait « explorer une approche intersectorielle visant à développer des interventions intégrées dans des territoires où se concentrent de multiples facteurs de défavorisation " (Cadre d'intervention, décembre 2001). C'est ainsi qu'est né un projet pilote dans le territoire de Montréal-Nord, un nouvel arrondissement de la ville de Montréal. Le but de l'intervention était de «mettre en œuvre une stratégie de concertation interministérielle au niveau régional afin de soutenir des projets locaux visant l'amélioration des conditions de vie des personnes démunies résidant dans les territoires ciblés ». Plus spécifiquement, on visait à «mettre de l'avant de nouvelles manières de faire, à établir de nouvelles solidarités entre les acteurs institutionnels locaux et régionaux, les intervenants sociaux et la communauté ». Nous présentons dans ce texte l'intervention qui se déroule à MontréalNord, son fonctionnement et ses processus, et nous tentons de répondre à la question suivante : cette façon de vivre la concertation fait-elle une différence dans les pratiques et dans l'atteinte des objectifs ?

\section{Pourquoi Montréal-Nord ?}

Montréal-Nord est une ancienne municipalité qui a été fusionnée à la ville de Montréal en 2002; elle constitue maintenant l'un des arrondissements de la ville. Une étude sur la défavorisation à Montréal ${ }^{2}$ a permis de faire ressortir, dans un premier temps, les territoires les plus touchés par la pauvreté sociale et économique. À l'aide de certains indicateurs de pauvreté tels la faible scolarité, les faibles revenus, le taux de chômage élevé, les ménages allouant plus de $30 \%$ de leur revenu au loyer, les familles monoparentales, l'aide sociale, Montréal-Nord est ressorti comme le deuxième quartier le plus défavorisé sur l'̂̂le de Montréal. Dans un deuxième temps, une analyse des dépenses publiques allouées à chacun des territoires sur le plan social a permis de faire ressortir des disparités importantes. Le territoire de Montréal-Nord a été choisi parce qu'il est marqué par une grande défavorisation ainsi qu'un ratio moins élevé des dépenses publiques.

\section{La démarche-action : structure et fonctionnement}

Pour atteindre les objectifs proposés, une structure fonctionnelle simple a été mise en place (figure 1). Elle est composée essentiellement d'un comité de pilotage régional-local, de deux comités de projets et d'un comité d'évaluation. Le comité de pilotage regroupe les acteurs essentiels de la démarche : représentants des instances régionales, représentants des institutions locales et représentants des groupes communautaires. Ce comité constitue la «plaque tournante» de la démarche.

Deux comités de projet coordonnent les actions principales de la démarche-action. Le projet « Soutien aux jeunes familles » vise à outiller et à accompagner les jeunes familles défavorisées pour lutter contre la pauvreté et sa perpétuation de génération en génération. Quant au projet «Un itinéraire pour tous », il vise à 
mobiliser la communauté du secteur Nord-Est pour améliorer son milieu de vie, notamment par la mise sur pied d'un centre socio-communautaire.

Ces projets ont été proposés par l'instance de concertation locale du mouvement des «Villes en santé ». Ils ont été élaborés par les acteurs locaux, et les partenaires régionaux se sont engagés à favoriser le financement des activités. Il y a très peu de nouveaux programmes destinés spécifiquement aux actions entreprises, mais des sommes importantes provenant de diverses sources sont canalisées dans ces projets.

Enfin, le comité d'évaluation accompagne la démarche de façon régulière. Avec l'approche participative et réflexive adoptée pour le devis, l'évaluation amène les participants à analyser constamment leur action et à la modifier s'il y a lieu.

Figure 1 - Organigramme fonctionnel

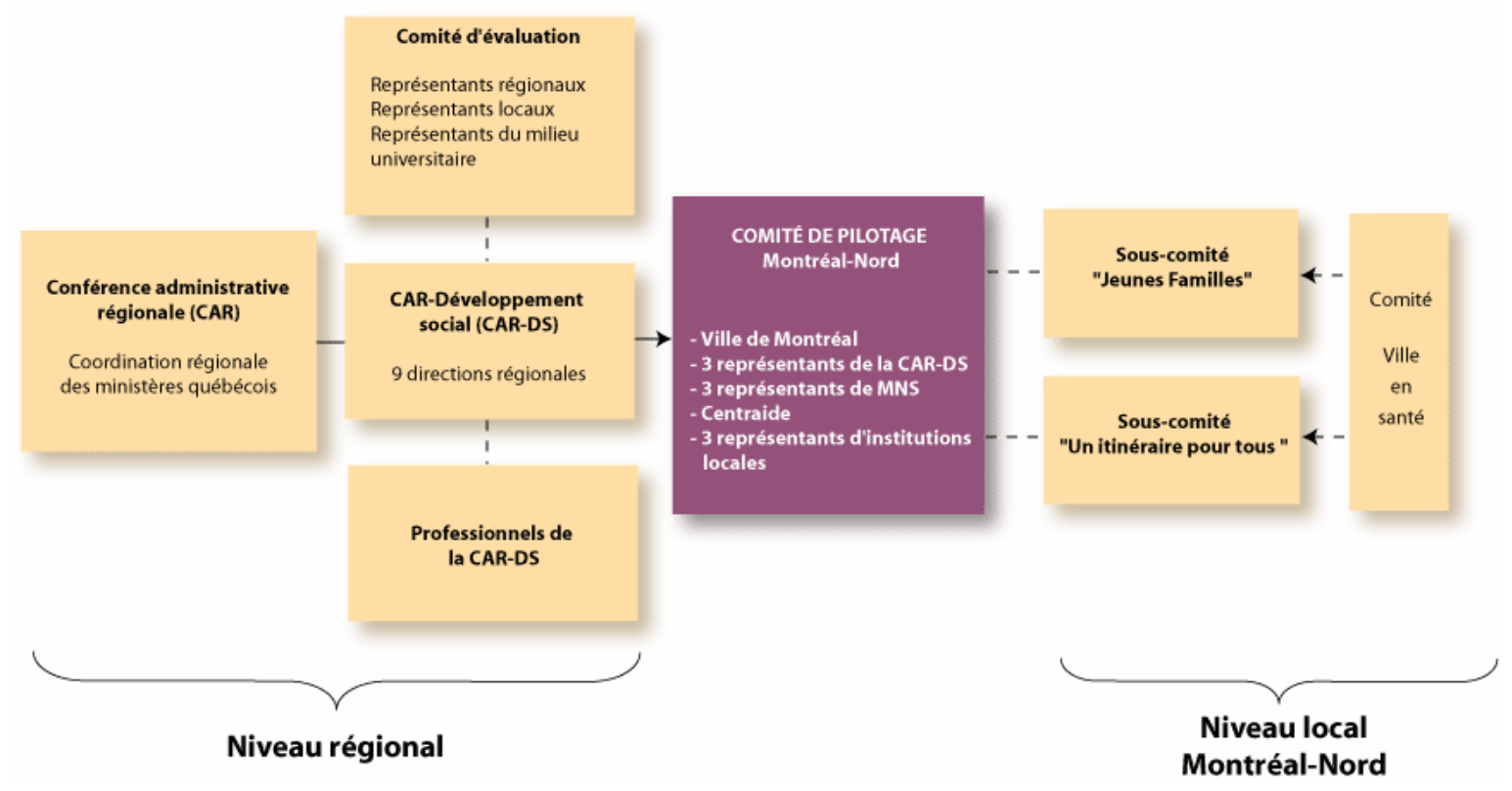

\section{Originalité de la démarche}

L'originalité de cette démarche ne réside pas tant dans les objectifs, qui ne sont pas nouveaux, ni dans les actions, mais plutôt dans l'approche et les processus. Cette approche vise à faciliter, harmoniser, lier, soutenir; elle se veut une "valeur ajoutée » à des éléments existants. Le suivi et la continuité sont majeurs; les processus pour implanter cette approche sont importants également.

L'élément central de la démarche est l'articulation entre le niveau local et le niveau régional. L'articulation se traduit dans un mécanisme de suivi, le Comité de pilotage régional-local. Le comité de pilotage est une instance pivot qui coordonne l'ensemble de la démarche. Ce comité favorise la concertation tant verticale, entre les instances régionales et les organismes locaux, que horizontale, entre les organismes locaux, communautaires et institutionnels. Les acteurs du ni- veau local et ceux des ministères bailleurs de fonds participent donc à la même instance, ce qui est nouveau. Les intervenants des ministères, à l'origine de la démarche, souhaitaient jouer un rôle plus actif que de simplement approuver des plans d'action et allouer des budgets; ils se considèrent donc " acteurs », d'une certaine façon, même si leur rôle n'en est pas un de premier plan.

L'approche transversale constitue un autre élément important de la démarche. Les acteurs ont adopté une façon nouvelle d'intervenir, mettant en lien divers programmes et services gouvernementaux dans l'optique d'une approche plus globale, ce qui ne se fait pas couramment. Au Québec, on a plutôt tendance à intervenir en « silo » (pas d'arrimage entre les services offerts). Cette approche considère l'ensemble des problématiques et vise à développer diverses actions interreliées. Enfin, l'ensemble de la communauté définit les priorités, alors que les organismes publics et les 
organismes communautaires tentent d'harmoniser leurs interventions. C'est une approche de développement de la communauté par la communauté ellemême, ce qu'on appelle en «bon français » inspiré de l'américain l'empowerment. Plutôt que de définir des programmes, on demande aux acteurs du milieu ce qu'ils souhaitent réaliser et on propose de les soutenir, de faciliter les choses en adaptant les programmes.

\section{Conditions de succès}

Certaines conditions ont facilité l'émergence de cette démarche et d'autres se sont ajoutées pour en assurer le succès. Au début, il y a eu convergences d'idées et d'événements. Le fait d'impliquer une instance de concertation comme « Montréal-Nord en santé » dans le choix des priorités a permis de s'inspirer d'une vision du quartier et de son développement relativement partagée par l'ensemble. De plus, à la suite de la fusion municipale, le Sommet de Montréal, précédé du sommet d'arrondissement, a permis de réunir les principaux acteurs de Montréal-Nord et d'identifier des priorités locales. Le leadership exercé par les responsables de la ville dans l'arrondissement et par des organismes du milieu dans les comités a favorisé le démarrage rapide des actions.

La nouveauté et la flexibilité de l'approche entrent aussi en ligne de compte. Ce qui a été appliqué, ce n'est pas un programme de subvention auquel les organismes auraient dû se conformer, mais plutôt une adaptation et un enrichissement de programmes existants. Ainsi, il n'y avait pas de budget disponible au début, sauf pour un coup de pouce à la planification. Le financement des projets s'est effectué au fur et à mesure de leur développement par des réorientations et des transferts. Cela évite la rigidité des programmes, mais c'est évidemment plus aléatoire.

Les liens et la continuité entre les éléments de la structure sont importants également. Il n'y a pas de hiatus entre le comité de pilotage, les comités de projets et le comité d'évaluation. Il n'y a pas de hiérarchie entre les comités; par exemple, il n'y a pas un comité directeur composé de décideurs, des comités d'action avec des acteurs différents et une équipe d'évaluation en parallèle avec des experts externes. Même si certaines personnes ne participent qu'à une seule instance, plusieurs autres participent à deux ou trois comités. Ces liens ne sont pas formels et l'information circule rapidement et librement de l'un à l'autre.

Tout cela est la conséquence ou la cause, ou les deux, d'un climat de confiance qui règne dans la démarche. Il existe une connivence, une complicité entre les acteurs qui incluent même les partenaires régionaux. Cela rejoint un mot de Gérard Divay, expert en revitalisation urbaine, qui croit que les acteurs d'une telle démarche doivent devenir des " conspirateurs », des gens qui s'entendent pour travailler ensemble étroitement et aller chercher les ressources dont ils ont besoin.

Enfin, une grande participation caractérise la démarche-action. Beaucoup de personnes participent aux quatre comités décrits ainsi qu'à plusieurs souscomités mis en place pour des activités spécifiques (près de 90 intervenants et citoyens). La participation est vécue à tous les niveaux, toutes les étapes, toutes les instances. Il en est de même pour les citoyens qui participent à des comités et qui se mobilisent particulièrement autour de l'un des deux projets d'intervention.

\section{C'est une approche de développement de la communauté par la communauté elle-même.}

\section{L’évaluation}

L'évaluation adopte le même modèle intersectoriel que l'ensemble de la démarche. Elle est gérée par un comité représentant les divers partenaires qui participent aux actions. Elle préconise une approche participative et réflexive qui se traduit par la participation des acteurs à la définition des outils et à leur utilisation sur une base régulière. Les objectifs poursuivis sont de quatre ordres :

- mise en place de l'approche participative;

- évaluation du processus de la démarche;

- évaluation du rôle de la CAR-DS comme acteur en développement social;

- évaluation des résultats. 
Les deux premiers volets ont été réalisés depuis un an, et le comité d'évaluation en est maintenant rendu à la phase d'évaluation des résultats.

\section{II importe de faire des liens avec les autres projets montréalais de revitalisation et de susciter la réflexion et le développement des connaissances sur ce type d'intervention territoriale concertée}

\section{Premiers résultats observés}

Après une année de fonctionnement, certaines constatations nous permettent d'estimer que des objectifs sont atteints :

- des budgets ont été affectés directement aux deux grands projets par diverses organisations (ministères, organismes publics, ville, secteur privé); à lui seul le bâtiment du centre socio-communautaire compte pour plus de 12 millions \$;

- d'autres budgets sectoriels régionaux sont venus soutenir les interventions (par exemple, en éducation, le projet « Éveil à la lecture »);

- les programmes ont été utilisés de façon plus souple pour s'adapter aux besoins locaux et ils ont été enrichis de volets complémentaires, comme d'autres activités auprès des jeunes familles;

- des liens se sont tissés entre les organismes communautaires, la ville, les organismes gouvernementaux et le privé;

- au niveau local, le milieu a appris à travailler autrement, et cette stratégie d'approche globale et de mise en réseau des ressources s'applique à d'autres projets (dans le logement par exemple);

- les interventions des ministères s'harmonisent au niveau régional, entre autres pour le choix des quartiers prioritaires d'intervention, et tendent à faire des liens entre les programmes appliqués;
- Montréal-Nord devient un territoire où s'expérimentent de nouvelles approches de lutte à la pauvreté en mobilisant, de façon concomitante, les décideurs de divers niveaux (régional, local).

\section{Conclusion}

Au cours de 2005, certains défis nous attendent :

- maintenir la cohésion du partenariat entre les organismes publics, communautaires et privés;

- accentuer l'harmonisation entre les directions régionales des ministères;

- améliorer les communications entre les divers groupes de travail;

- conserver une démarche évolutive et éviter d'en faire un programme institutionnalisé;

- évaluer les impacts réels sur la population et l'organisation des services ainsi que l'atteinte des objectifs;

- et, le plus ardu, poursuivre la démarche malgré un contexte politique en changement, sinon en bouleversement.

Comme la « Démarche-action » s'inscrit dans la mouvance de la revitalisation urbaine en cours présentement au Québec, il importe également de faire des liens avec les autres projets montréalais de revitalisation et de susciter la réflexion et le développement des connaissances sur ce type d'intervention territoriale concertée.

\section{Note et référence}

Danielle Aveline est conseillère au Ministère des Affaires municipales et des Régions; André Bergeron est agent de planification, Développement social et Liaison municipale, à la Direction de la santé publique.

2 MAMM (2001). Portrait de la défavorisation et des investissements sur le territoire de la nouvelle Ville de Montréal, mai. 